\title{
Linear negative dispersion with a gain doublet via optomechanical interactions
}

\author{
Jiayi Qin, * Chunnong Zhao, Yiqiu Ma, Li Ju, and David G. Blair \\ School of Physics, University of Western Australia, Western Australia 6009, Australia \\ ${ }^{*}$ Corresponding author: jiayiqinphysics@gmail.com \\ Received February 25, 2015; accepted April 13, 2015; \\ posted April 23, 2015 (Doc. ID 235138); published May 11, 2015
}

\begin{abstract}
Optical cavities containing a negative dispersion medium have been proposed as a means of improving the sensitivity of laser interferometric gravitational wave detectors through the creation of white-light signal recycling cavities. Here we demonstrate that negative dispersion can be realized using an optomechanical cavity pumped by a blue detuned doublet. We used an 85-mm cavity with an intracavity silicon nitride membrane. Tunable negative dispersion is demonstrated, with a phase derivative $d \varphi / d f$ from $-0.14 \mathrm{Deg} \cdot \mathrm{Hz}^{-1}$ to $-4.2 \times 10^{-3} \mathrm{Deg} \cdot \mathrm{Hz}^{-1}$. (C) $2015 \mathrm{Optical}^{\circ}$ Society of America

OCIS codes: (120.4880) Optomechanics; (260.2030) Dispersion.

http://dx.doi.org/10.1364/OL.40.002337
\end{abstract}

In 1839, Hamilton pointed out that the group velocity of a wave is different from its phase velocity [1]]. In 1881, Lord Rayleigh [2] commented that a pulse of light travels at the group velocity (rather than the phase velocity) inside a medium. He further developed the theory of anomalous dispersion and first demonstrated anomalous dispersion for a mechanical oscillator [3, $\underline{4}]$. Later, Sommerfeld and Brillouin theoretically showed that dispersion is anomalous inside an absorption line, where the group velocity exceeds $c$, the light velocity in the vacuum [5].

More recently, anomalous dispersion has been investigated in various schemes. In 1970, Garrett and McCumber [6] studied the properties of Gaussian light pulses in absorptive media. In 1971, Casperson and Yariv [7] demonstrated that the velocity of ultrashort pulses through a high-gain medium is a function of the gain, and agrees with the theoretical results. In 1981, Chu and Wong [8] experimentally verified the theoretical predictions in [ㅁ] showing that pulses propagating in samples of GaP:N tuned to the bound A-exciton line propagate with little pulse shape distortion, at a group velocity that either exceeds $c$ or becomes negative. In 1985, Segard and Macke [9] demonstrated significant pulse advances $\sim 2 \mu$ s with negligible distortion through a linear molecular absorber at millimeter wavelengths.

More recently, negative dispersion was observed in atomic systems via electromagnetic interactions. The effect is caused by atomic coherence in degenerate two-level systems, which leads to negative dispersion at the resonant frequency of an atomic transition. In 1999, Akulshin and Chiao [10] realized steep negative dispersion up to $d n / d \nu \simeq-6 \times 10^{-11} \mathrm{~Hz}^{-1}$ in coherently prepared $\mathrm{Rb}$ vapor.

In 1994, Steinberg and Chiao [10] realized that in a medium with a gain doublet there exists a transparent region with an anomalous dispersion between the two gain peaks. In 2000, Wang et al. [11] reported gainassisted linear negative dispersion in atomic cesium gas. In 2011, Safavi-Naeini et al. [12] reported an optically tunable delay of $50 \mathrm{~ns}$ and superluminal light with a $1.4 \mathrm{~ms}$ signal advance in a nanoscale optomechanical crystal. In 2012, Ivanov et al. [ $\underline{13}]$ observed the "fast light" effect in a cryogenic microwave resonator.
The concept of using a negative dispersion medium to improve the broadband sensitivity of gravitational wave (GW) detectors was first proposed by Wicht et al. [14] in 1997. In 2007, Salit and Shahriar [15] proposed a practical design for a white-light signal recycling GW interferometer by putting a negative dispersion medium inside the recycling cavity. By taking into account the quantum noise and stability of the system, Ma et al. [16] excluded the possibility of using such a double-pumped optomechanical filter for improving the shot-noise-limited sensitivity of GW detectors in the stable regime. In order to achieve sensitivity improvement, one approach is exploring the unstable regime with additional feedback control for stabilization, as proposed by Miao et al. [17]. The other approach is using multiple control fields, instead of just two, to construct an optical filter that has a different spectral shape, e.g., the one considered by Zhou et al. [18] but using atomic systems.

In this Letter, we make a proof-of-principle demonstration of using the optomechanical device to realize an active filter that possesses interesting optical properties. We report the experimental realization of a gain-assisted linear negative dispersion in an optomechanical system with two blue-detuned control beams. Using an 85-mm optical cavity coupled with a silicon nitride membrane we demonstrate optically tunable negative dispersion that is equivalent to replacing the cavity with a negative dispersion medium that has a phase derivative $d \varphi / d f$ from $-0.14 \mathrm{Deg} \cdot \mathrm{Hz}^{-1}$ to $-4.2 \times 10^{-3} \mathrm{Deg} \cdot \mathrm{Hz}^{-1}$. Our result shows the potential possibility of using such optomechanical systems to build white-light signal recycling cavities in laser interferometric GW detectors for improving the sensitivity subject to the significant reduction of the mechanical resonator's thermal noise. Such a freespace optomechanical filter can be an alternative but less lossy compared to atomic systems where the light has to go through the medium [16-19]. Our demonstration shows the feasibility of using optomechanical interactions to achieve a required optical response.

Optomechanical interactions can be used to create linear negative dispersion in the scheme shown in Fig. 1. Two control beams maintain strong fields $\bar{a}_{ \pm} e^{-i\left(\omega_{p} \pm \delta_{0}\right) t}$ in the cavity at the resonant frequency $\omega_{0}$. A weak signal 


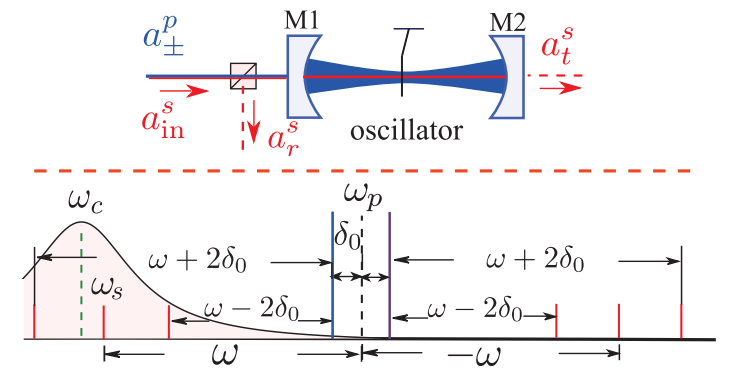

Fig. 1. Configuration schematics (top) and frequency relationships. The signal light having frequency $\omega_{s}$ is injected into an optical cavity with a silicon nitride membrane in the middle that acts as an oscillator at the resonant frequency $\omega_{m}$. The resonant frequency of the coupled cavity is $\omega_{c}$. The position of the membrane is chosen to introduce a linear optomechanical coupling. The radiation pressure forces from the beatings between the signal light at the frequency $\omega_{s}=\omega_{p}-\omega$ and the control beams at the frequency $\omega_{p} \pm \delta_{0}$ drive the mechanical oscillator which in turn creates sidebands destructively interfering with the signal light, which in effect creates linear negative dispersion.

light $\delta \hat{a}_{\text {in }}=\hat{a}_{\text {in }}^{s} e^{-i \omega_{s} t}$ is injected into the same port (M1). The frequency differences between the control field's central frequency $\omega_{p}$ and the signal fields need to be close to the mechanical resonant frequency for effective driving of the mechanical mode.

The Hamiltonian that describes this system is given by

$$
\hat{H}=\hbar\left(\omega_{0}+g_{0} \hat{x}\right) \hat{a}^{\dagger} \hat{a}+\hat{H}_{m}+\hat{H}_{\gamma} .
$$

Here, $\hat{H}_{m}=\hat{p}^{2} / 2 m+m \omega_{m}^{2} \hat{x}^{2} / 2$ is the free Hamiltonian of the mechanical oscillator. $H_{\gamma}=-i \hbar \sqrt{2 \gamma_{1}} \hat{a} \hat{a}_{\text {in }}^{\dagger}-$ $i \hbar \sqrt{2 \gamma_{2}} \hat{a} \hat{b}_{\text {in }}^{\dagger}+$ h.c describes the interaction between the intracavity field $\hat{a}$ and external electromagnetic fields $\hat{a}_{\text {in }}$ and $\hat{b}_{\text {in }}$ with bandwidths $\gamma_{1}=c T_{1} / 4 L$ and $\gamma_{2}=$ $c T_{2} / 4 L$ through the cavity mirrors $\mathrm{M} 1$ and $\mathrm{M} 2$, respectively. $g_{0}$ is the linear optomechanical coupling strength. Since the signal light $\hat{a}_{\text {in }}^{s}(\omega)$ in our experiment is a classical field, we neglect the vacuum fluctuation term and $\hat{b}_{\text {in }}$. In the rotating frame at frequency $\omega_{p}$, where $\omega=\omega_{p}-\omega_{s}$, we have

$$
\begin{aligned}
\hat{x}\left(\omega_{ \pm}\right)= & -\chi_{m}^{-1}\left(\omega_{ \pm}\right) \hbar g_{0}\left[\bar{a}_{\mp} \hat{a}^{\dagger}\left(\omega \pm 2 \delta_{0}\right)+\bar{a}_{ \pm} \hat{a}^{\dagger}(\omega)\right. \\
& \left.+\bar{a}_{+}^{*} \hat{a}(-\omega)+\bar{a}_{ \pm}^{*} \hat{a}\left(-\omega \mp 2 \delta_{0}\right)\right], \\
\hat{a}(\omega)= & \chi_{c}^{-1}(\omega)\left\{-i g_{0}\left[\bar{a}_{+} \hat{x}\left(\omega_{+}\right)+\bar{a}_{-} \hat{x}\left(\omega_{-}\right)\right]\right. \\
& \left.+\sqrt{2 \gamma_{1}} \hat{a}_{\mathrm{in}}^{s}(\omega)\right\},
\end{aligned}
$$

where $\omega_{ \pm}=\omega \pm \delta_{0}$, and $\chi_{m}\left(\omega_{ \pm}\right)=m\left(\omega_{m}^{2}-\omega_{ \pm}^{2}-i \gamma_{m} \omega_{ \pm}\right)$ and $\chi_{c}(\omega)=[-i(\omega-\Delta)+\gamma]$ are the mechanical and optical susceptibility, respectively. The $\gamma_{m}$ and $\gamma=\gamma_{1}+\gamma_{2}$ are the bandwidths of the mechanical oscillator and the cavity, respectively. We choose the frequency detuning to be $\Delta=\omega_{p}-\omega_{c} \sim \omega_{m}$. In our system where $\delta_{0} \ll \gamma$ we make use of the near-resonance approximations: $\omega-\Delta \ll \gamma, \omega-\Delta \pm 2 \delta_{0} \ll \gamma$. Thus, the optical susceptibilities can be approximately written as $\chi_{c}\left(\omega \pm 2 \delta_{0}\right) \sim$ $\chi_{c}(\omega) \sim \gamma$.
Since the optical anti-Stokes sidebands $\hat{a}(-\omega)$ and $\hat{a}\left(-\omega \mp 2 \delta_{0}\right)$ are far detuned where $\gamma \ll 2 \omega_{m}$, they can be safely ignored. This approximation is called resolved sideband approximation. Here, the Stokes sidebands under the above near-resonance approximation can be written as

$$
\begin{gathered}
\hat{a}^{\dagger}(\omega) \approx \frac{i g_{0}}{\gamma}\left[\bar{a}_{+}^{*} \hat{x}\left(\omega_{+}\right)+\bar{a}_{-}^{*} \hat{x}\left(\omega_{-}\right)\right]+\frac{\sqrt{2 \gamma_{1}}}{\gamma} \hat{a}_{\mathrm{in}}^{s \dagger}(\omega), \\
\hat{a}^{\dagger}\left(\omega \pm 2 \delta_{0}\right) \approx \frac{i g_{0}}{\gamma} \bar{a}_{+}^{*} \hat{x}\left(\omega_{ \pm}\right) .
\end{gathered}
$$

Substituting Eqs. (3a) and (3b) into the radiation pressure force terms in the equations of motion for mechanical displacement, Eq. (2a) leads to

$$
\left[\begin{array}{cc}
\chi_{\mathrm{eff}}\left(\omega_{+}\right) & i \sqrt{\Gamma_{+} \Gamma_{-}} \\
i \sqrt{\Gamma_{+} \Gamma_{-}} & \chi_{\mathrm{eff}}\left(\omega_{-}\right)
\end{array}\right] \times\left[\begin{array}{c}
x\left(\omega_{+}\right) \\
x\left(\omega_{-}\right)
\end{array}\right]=\left[\begin{array}{c}
\beta_{+} \\
\beta_{-}
\end{array}\right] a_{\mathrm{in}}^{s^{\dagger}}(\omega) .
$$

The optomechanical antidamping rates $\Gamma_{\mp}$ are given by

$$
\Gamma_{ \pm}=\frac{\hbar g_{0}^{2}\left|\bar{a}_{ \pm}\right|^{2}}{2 m \omega_{m} \gamma}
$$

and can be tuned by changing the control field power. The effective mechanical response function is $\chi_{\text {eff }}\left(\omega_{ \pm}\right)=$ $\chi_{m}\left(\omega_{ \pm}\right) / 2 m \omega_{m}+i\left(\Gamma_{+}+\Gamma_{-}\right)$, where in the near-resonance case we have $\chi_{m}(\omega \pm) \approx-2 m \omega_{m}\left(\omega_{ \pm}-\omega_{m}+i \gamma_{m}\right)$. The parameters $\beta_{ \pm}$are defined as $\beta_{ \pm} \equiv-\sqrt{2 \gamma_{1}} \Gamma_{ \pm} / g_{0} \bar{a}_{ \pm}^{*}$. The beat between the signal field and one control field $\bar{a}_{+}$creates a radiation pressure force that induces the mechanical motion $x\left(\omega_{+}\right)$. This mechanical motion $x\left(\omega_{+}\right)$then modulates the control fields $\hat{a}_{+}$and $\hat{a}_{-}$to produce Stokes sidebands at frequency $\omega$ and $\omega+2 \delta_{0}$, respectively. The antidamping rates $\Gamma_{+}$and $\Gamma_{-}$in $\chi_{\text {eff }}\left(\omega_{+}\right)$are caused, respectively, by the beat between $\hat{a}(\omega)$ and the control field $\bar{a}_{+}$and between $\hat{a}\left(\omega+2 \delta_{0}\right)$ and the control field $\bar{a}_{-}$. The field at frequency $\omega$ also interacts with the control field $\hat{a}_{-}$to create a radiation pressure force. This force drives the mechanical motion $x\left(\omega_{-}\right)$and gives rise to the interaction terms $\sqrt{\Gamma_{+} \Gamma_{-}}$ in Eq. (4). The terms on the bottom line of Eq. (4) are similarly explained.

Substituting Eq. (4) into Eq. (3a) and using the inputoutput relations $\hat{a}_{\mathrm{r}}^{\dagger}=-\hat{a}_{\mathrm{in}}^{\dagger}+\sqrt{2 \gamma_{1}} \hat{a}^{\dagger}$ and $\hat{a}_{\mathrm{t}}^{\dagger}=\sqrt{2 \gamma_{2}} \hat{a}^{\dagger}$, we have the effective reflectivity and transmissivity as

$$
\begin{gathered}
r(\omega)=-1+2 \eta_{c} \frac{1}{\left(1-i \chi_{\varphi}\right)}, \\
t(\omega)=2 \sqrt{\left(1-\eta_{c}\right) \eta_{c}} \frac{1}{\left(1-i \chi_{\varphi}\right)},
\end{gathered}
$$

where $\quad \chi_{\varphi}=\Gamma_{+} /\left[\omega-\omega_{m}+\delta_{0}+i \gamma_{m}-i \Gamma_{-}\right]+\Gamma_{-} /[\omega-$ $\left.\omega_{m}-\delta_{0}+i \gamma_{m}-i \Gamma_{+}\right]$and the cavity coupling parameter is $\eta_{c}=\gamma_{1} /\left(\gamma_{1}+\gamma_{2}\right)$. In Fig. 2 , we give the theoretical reflectivity and transmissivity using parameters that are matched to our measurement results in Fig. 4. In our experiment, we detect the signals at the transmission port. To build white-light signal recycling cavities in GW interferometers, the reflection output will be used instead of 


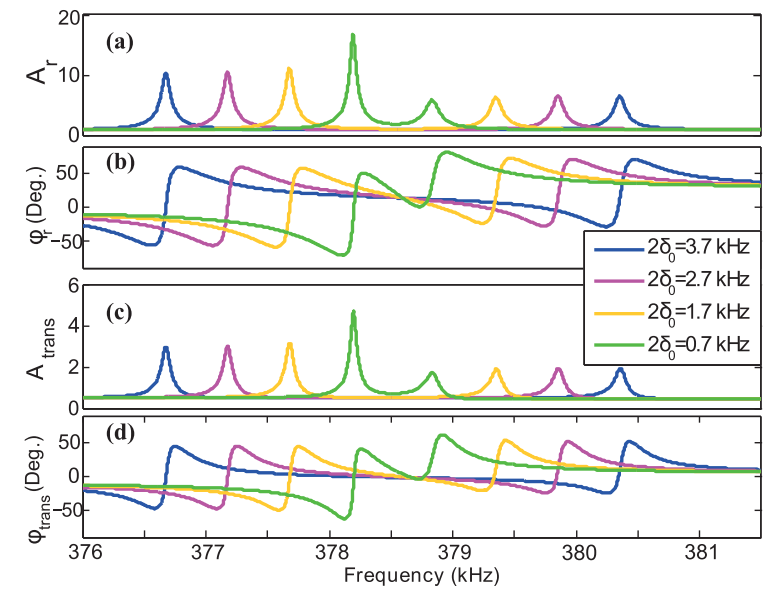

Fig. 2. Theoretical reflectivity and transmissivity in the double gain scheme: (a) reflectivity amplitude $A_{r}$; (b) reflectivity phase $\varphi_{r}$; (c) transmissivity amplitude $A_{\text {trans }}$; and (d) transmissivity phase $\varphi_{\text {trans }}$. The parameters are: $\gamma_{m}=340 \mathrm{~Hz}, \Gamma_{+}=$ $170 \mathrm{~Hz}, \Gamma_{-}=140 \mathrm{~Hz}, \omega_{m}=378.55 \mathrm{kHz}$, and the frequency detuning $\Delta-\omega_{m}=9 \mathrm{kHz}$ in $\chi_{c}(\omega)$.

the transmission one to reduce quantum noises coupled from the transmission port.

In our experimental setup shown in Fig. 3, the key element is an 85-mm high-finesse optical cavity containing a high-stress silicon nitride membrane with a quality factor of $\sim 1.5 \times 10^{6}$ at its fundamental mechanical resonance $\sim 378.5 \mathrm{kHz}$, measured at a vacuum level of $10^{-5}$ mbar [20]. The experiment was conducted at a relatively low vacuum $\left(\sim 10^{-2}\right.$ mbar $)$ to use the gas damping to stabilize the system since the blue detuned control light will create negative damping. The $Q$-factor was reduced to $\sim 10^{3}$. However, active or passive cooling can be used to stabilize the system and to suppress thermal noise in future practical systems.

Our optical cavity is mounted on an invar spacer machined by electrical discharge machining with accuracy

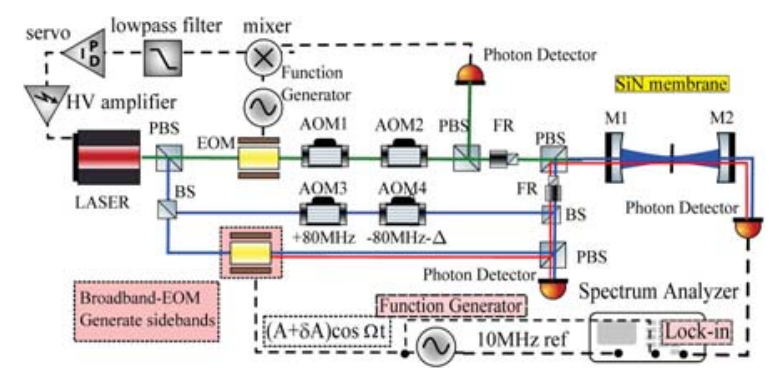

Fig. 3. Experimental setup. The $85 \mathrm{~mm}$-long cavity sits in a vacuum chamber with a central silicon nitride membrane oscillator $(1 \mathrm{~mm} \times 1 \mathrm{~mm} \times 50 \mathrm{~nm}$, effective mass $40 \mathrm{ng}$, index of refraction $n=2+2.5 \times 10^{-5} i$ ) [20]. The green line represents the locking light for stabilizing the laser frequency to the cavity resonance using Pound-Drever-Hall (PDH) locking [21]. The blue lines represent the control fields, with polarization orthogonal to the locking light. The broadband EOM generates a lower-sideband from the control light, which is our signal light (red line) with input power $\sim 0.2 \mathrm{~mW}$. The frequency differences between the locking and control fields were created using pairs of $\pm 80 \mathrm{MHz}$ acousto-optical modulators in the optical paths. BS and PBS are the beam splitter and polarized beam splitter, and FR is the faraday rotator.
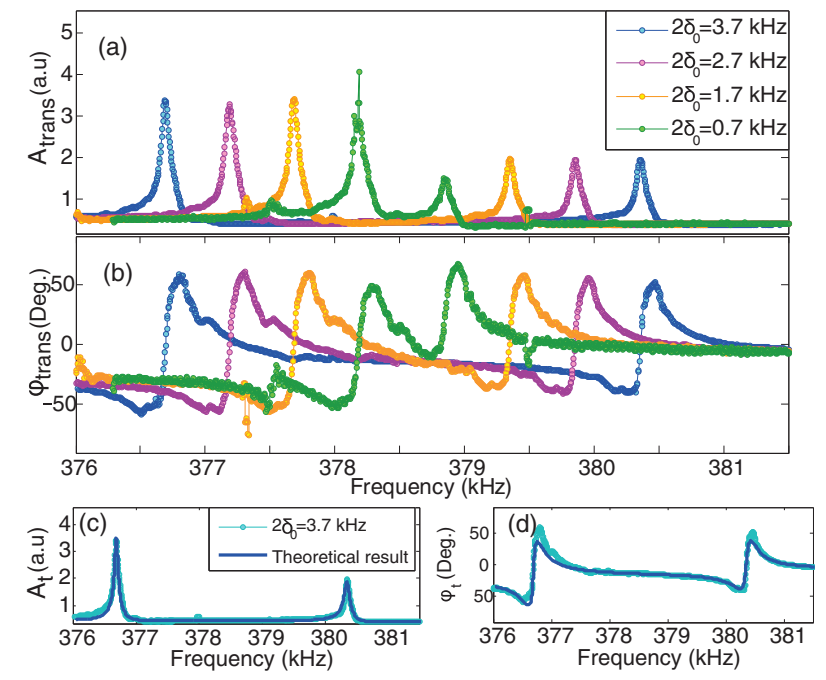

Fig. 4. Experimental transmissivity in the double gain scheme: (a) $A_{\text {trans }}$; (b) $\varphi_{\text {trans }}$; (c) and (d) combine transmissivity data and theoretical result at $2 \delta_{0}=3.7 \mathrm{kHz}$. The parameters are the same as in Fig. $\underline{2}$.

of $0.1 \mu \mathrm{m}$ and fixed in a vibration isolated vacuum tank. The mirrors M1 and M2 are clamped at the ends of the spacer. The membrane frame is bonded onto a piezoelectric actuator with Yacca gum, a natural resin with low intrinsic loss [22]. The transmissivity $T_{1}\left(T_{2}\right)$ of M1 (M2) is $245.1 \pm 2.8 \mathrm{ppm}(16.93 \pm 0.20 \mathrm{ppm})$, which was measured in an empty cavity. The experiment was conducted at room temperature using a $1064 \mathrm{~nm} \mathrm{Nd:YAG}$ laser [20].

To create a wide linear negative dispersive region, two blue-detuned control fields at frequencies $\omega_{p} \pm \delta_{0}$ were injected into the cavity. The field $\hat{a}_{-}$passes through a broadband electro-optic modulator (EOM) to generate the weak signal light $\hat{a}_{\text {in }}^{s}$. Using a lock-in amplifier [20], we measured the transmission of the cavity by detecting the beat signals between the signal field and the control fields at the transmission port. In Fig. 4 , we show experimental results of the transmission amplitude and phase for different frequency offsets $\delta_{0}$. In Fig. 5(a), we show the linear region of the transmission phase for frequency
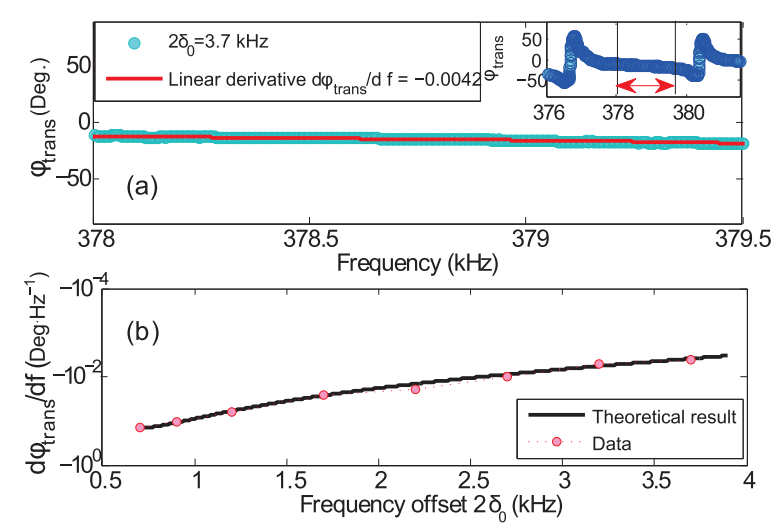

Fig. 5. (a) Zoomed-in linear transmission phase for frequency offset $\delta_{0}=3.7 \mathrm{kHz}$, and (b) linear derivatives of the transmissivity phase versus frequency offset $2 \delta_{0}$ at the frequency $\omega_{m}-0.28 \mathrm{kHz}$. 
offset $\delta_{0}=3.7 \mathrm{kHz}$ with linear curve fitting. While there are some small distortions in the phase response at large frequency offsets, the linear negative dispersion region is well behaved within expected error.

In Fig. 5(b), we show the first-order derivatives of the transmission phase at different frequency offsets, which range from $-0.14 \mathrm{Deg} \cdot \mathrm{Hz}^{-1}$ to $-4.2 \times 10^{-3} \mathrm{Deg} \cdot \mathrm{Hz}^{-1}$.

Tunable negative dispersion has been created in an optomechanical cavity with an intracavity silicon nitride membrane. Double pumping is used to create a wide linear negative dispersion regime. The system has similar performance to an atomic gas system with a gain doublet and could be a less lossy alternative. The technique described here can be extended to broadband sensitivity improvement using double-gain blue-detuned cavities with feedback control [17]. Practical systems would require a mechanical resonator with a high $Q$-factor to environment temperature ratio, as discussed in [17]. Optical dilution can be used to increase the mechanical $Q$-factor by a factor of a hundred or even more [23]. Our demonstration illustrates the potential of using optomechanical interactions to achieve the required active optical filter response for improving the sensitivity of GW detectors.

We thank Haixing Miao, Xu Chen, Yanbei Chen, and Stefan Danilishin for useful discussions. We thank Gary Light for technical support. We would like to thank the LIGO Scientific Collaboration, the Gingin International Advisory Committee, and our collaborators for useful advice. This research was supported by the Australian Research Council (DP120100898 and DP120104676).

\section{References}

1. W. R. Hamilton, Proc. R. Irish Acad., Sect. A 1, 267 (1839).

2. Rayleigh, Nature XXIV, 382 (1881).

3. Rayleigh, Nature LX, 64 (1899).
4. Rayleigh, Philos. Mag. 48(290), 151 (1899).

5. L. Brillouin, Wave Propagation and Group Velocity (Academic, 1960).

6. C. G. B. Garrett and D. E. McCumber, Phys. Rev. A 1, 305 (1970).

7. L. Casperson and A. Yariv, Phys. Rev. Lett. 26, 293 (1971).

8. S. Chu and S. Wong, Phys. Rev. Lett. 48, 738 (1982).

9. B. Segard and B. Macke, Phys. Lett. 109A, 213 (1985).

10. A. M. Steinberg and R. Y. Chiao, Phys. Rev. A 49, 2071 (1994).

11. L. J. Wang, A. Kuzmich, and A. Dogariu, Nature 406, 277 (2000).

12. A. H. Safavi-Naeini, T. P. Mayer Alegre, J. Chan, M. Eichenfield, M. Winger, Q. Lin, J. T. Hill, D. E. Chang, and O. Painter, Nature 472, 69 (2011).

13. E. Ivanov, J. Bourhill, D. Creedon, J. Hartnett, and M. Tobar, J. Phys. B 45, 221001 (2012).

14. A. Wicht, K. Danzmann, M. Fleischhauer, M. Scully, G. Müller, and R. H. Rinkleff, Opt. Commun. 134, 431 (1997).

15. M. Salit and M. S. Shahriar, J. Opt. 12, 104014 (2010).

16. Y. Ma, H. Miao, C. Zhao, and Y. Chen, "Quantum noise of white light cavity using double-pumped gain medium," arXiv:1501.01349 (2015) [quant-ph].

17. H. Miao, Y. Ma, C. Zhao, and Y. Chen, "Enhancing detection bandwidth by using unstable optomechanical filters," https://dcc.ligo.org/P1400255.

18. M. Zhou, Z. Zhou, and S. M. Shahriar, "Quantum noise limits in white-light-cavity-enhanced gravitational wave detectors," arXiv:1410.6877 (2014) [gr-qc].

19. E. E. Mikhailov, K. Goda, T. Corbitt, and N. Mavalvala, Phys. Rev. A 73, 053810 (2006).

20. J. Qin, C. Zhao, Y. Ma, X. Chen, L. Ju, and D. G. Blair, Phys. Rev. A 89, 041802(R) (2014).

21. E. D. Black, Am. J. Phys. 69, 79 (2001).

22. S. W. Schediwy, S. Gras, L. Ju, and D. G. Blair, Rev. Sci. Instrum. 76, 026117 (2005).

23. Y. Ma, S. L. Danilishin, C. Zhao, H. Miao, W. Z. Korth, Y. Chen, R. Ward, and D. G. Blair, Phys. Rev. Lett. 113, 151102 (2014). 